\title{
ANALYSIS OF A SPLITTING SCHEME FOR A CLASS OF RANDOM NONLINEAR PARTIAL DIFFERENTIAL EQUATIONS *
}

\author{
Romain DuboscQ ${ }^{1}$ And Renaud Marty ${ }^{2}$
}

\begin{abstract}
In this paper, we consider a Lie splitting scheme for a nonlinear partial differential equation driven by a random time-dependent dispersion coefficient. Our main result is a uniform estimate of the error of the scheme when the time step goes to 0. Moreover, we prove that the scheme satisfies an asymptotic-preserving property. As an application, we study the order of convergence of the scheme when the dispersion coefficient approximates a (multi)fractional process.
\end{abstract}

Mathematics Subject Classification. 35Q55, 65M15, 60H15, 60F17.

Received May 4, 2015. Revised March 11, 2016. Accepted September 16, 2016.

\section{INTRODUCTION}

The study of partial differential equations (PDEs) driven by random processes is a subject of much interest because of their numerous applications. In particular, Schrödinger type equations with random coefficients are relevant models for wave propagation, Bose-Einstein condensates, or optical fibers for instance. The driving random processes or random coefficients can model random perturbations of physical quantities, inhomogeneities of the medium (wave propagation), or a random dispersion coefficient (optical fibers). They can be defined in terms of Brownian motion (for mixing properties) or fractional Brownian motion (for long-range properties).

Besides applications, the asymptotic analysis and numerical simulations of such equations are crucial questions. Since the splitting schemes are quite simple to implement, they are often used for the simulation of nonlinear evolution PDEs $[6,16,32]$. They consist of constructing a numerical solution by combining the solutions of two partial problems which can be solved explicitly and obtained by an operator splitting. These methods are consistent for deterministic nonlinear Schrödinger equations [6] and can take different forms (mainly the Lie and Strang schemes). For instance, in [6] the authors prove that the Lie scheme is of order 1 and the Strang scheme is of order 2 (in dimension 1 or 2). The Lie scheme has also been studied for nonlinear Schrödinger equations with random white-noise dispersion [27]. In this case, it is proven that the Lie scheme has an order of convergence bounded below by $1 / 2$.

\footnotetext{
Keywords and phrases. Nonlinear partial differential equations, splitting, stochastic partial differential equations, asymptotic-Preserving schemes, fractional and multifractional processes.

* This work was partially supported by the French ANR grants MICROWAVE NT09_460489 (http: //microwave. math. cnrs. $\mathrm{fr} /$ ) and BECASIM ANR-12-MONU-0007-02 (http://becasim.math.cnrs.fr/).

1 Institut Mathématique de Toulouse, 118 route de Narbonne, 31062 Toulouse, cedex, France.

romain. duboscq@math. univ-toulouse. fr

2 Université de Lorraine, CNRS, Institut Elie Cartan de Lorraine, UMR 7502, Vandoeuvre-lès-Nancy, 54506, France.

renaud.marty@univ-lorraine.fr
} 
In addition to convergence, a property of great importance for a numerical scheme is the asymptoticpreserving property. It can be described as follows. Let $\left\{u^{\varepsilon}\right\}_{\varepsilon \geq 0}$ be the solutions of a family of problems such that $\lim _{\varepsilon \rightarrow 0} u^{\varepsilon}=u^{0}$, and $\left\{u^{\varepsilon, h}\right\}_{\varepsilon \geq 0}$ the family of numerical solutions approximating $\left\{u^{\varepsilon}\right\}_{\varepsilon \geq 0}$ for a time-step $h>0$ and obtained from a numerical scheme. We say that the given numerical scheme is asymptotic-preserving if $u^{\varepsilon, h}$ approximates $u^{\varepsilon}$ independently of $\varepsilon$ and $\lim _{\varepsilon \rightarrow 0} u^{\varepsilon, h}=u^{0, h}$. There exists a lot of works dealing with Asymptotic-Preserving (AP) property in various problems (for instance $[4,10,13,19,23,25]$ ). In particular for splitting schemes for Schrödinger and/or random equations, we mention for instance $[2,3,7,21,27]$.

In this paper we consider a nonlinear PDE driven by a general random process with continuous sample paths. We analyze a Lie splitting scheme for this equation. We prove that the scheme converges and we establish a uniform error bound in terms of the sample paths of the driving process. The form of this error bound and a continuity theorem are then used to establish a general asymptotic-preserving property for the scheme. These results are then applied to nonlinear PDEs driven by processes approximating fractional and multifractional processes. This generalizes results of [27] dealing with processes approximating a Brownian motion.

In Section 2, we introduce the setting and study the order of convergence of the splitting scheme and we establish the AP property and study equations driven by processes approximating (multi)fractional processes in Section 3. Section 4 is dedicated to the proofs of the results of Section 2.

\section{Notation}

For a measurable space $E$ and a normed space $F$, we denote by $L^{2}(E, F)$ the space of the square integrable functions from $E$ to $F$. For the sake of clearness, we denote by $L^{2}$ the space $L^{2}(\mathbb{R}, \mathbb{C})$. For every $p \in \mathbb{N}^{*}$, we introduce $H^{p}$ as the Sobolev space of the square integrable functions from $\mathbb{R}$ to $\mathbb{C}$ such that the first $p$ derivatives are square integrable. We consider $\|\cdot\|_{L^{2}},\|\cdot\|_{H^{1}},\|\cdot\|_{H^{2}}, \ldots$, as their associated norms. For every function $v \in L^{2}$, we denote the Fourier transform of $v$ by $\mathcal{F}(v)$ or $\mathcal{F}_{x}(v(x))$ : for every $\xi \in \mathbb{R}$,

$$
\mathcal{F}(v)(\xi)=\mathcal{F}_{x}(v(x))(\xi)=\frac{1}{\sqrt{2 \pi}} \int_{\mathbb{R}} \mathrm{e}^{i x \xi} v(x) \mathrm{d} x .
$$

We recall that $\|v\|_{L^{2}}=\|\mathcal{F}(v)\|_{L^{2}}$ and if $v \in H^{1}$, then $\mathcal{F}_{x}\left(i v^{\prime}(x)\right)(\xi)=\xi \mathcal{F}_{x}(v(x))(\xi)$. For every function $w \in L^{2}$, we denote the inverse Fourier transform of $w$ by $\mathcal{F}^{-1}(w)$ or $\mathcal{F}_{\xi}^{-1}(w(\xi))$.

Throughout the paper, all the random variables are defined on a probability space $(\Omega, \mathcal{T}, \mathbb{P})$, the corresponding expectation being $\mathbb{E}$.

When considering a Lipschitz function $g$, we designate by $\|g\|_{L i p}$ its Lipschitz constant.

Finally, for every $k \in \mathbb{N}^{*}$, we denote by $\mathcal{B}_{k}$ the set of functions $g: \mathbb{R} \rightarrow \mathbb{R}$ such that $g$ is $k$ times differentiable and $g^{\prime}, g^{(2)}, \ldots$, and $g^{(k)}$ are bounded.

\section{MAIN RESULtS}

\subsection{Nonlinear PDE with random dispersion}

Let $t_{0}$ and $T$ such that $0<t_{0}<T<\infty$. We consider the following nonlinear PDE with random dispersion written in differential form:

$$
u(t, x)=u_{t_{0}}(x)+i \int_{t_{0}}^{t} P\left(i \frac{\partial}{\partial x}\right) u(\theta, x) \circ \mathrm{d} W(\theta)+\int_{t_{0}}^{t} g(u(\theta, x)) \mathrm{d} \theta, \quad(t, x) \in\left[t_{0}, T\right] \times \mathbb{R} .
$$

The function $u_{t_{0}}$ is the initial condition at $t_{0}$. The function $g$ is the nonlinear function such that $g(0)=0$ and whose other assumptions we precise later. The notation $W$ designates a continuous stochastic process, which can be eventually a deterministic function; $P$ is a polynomial with real coefficients and its degree is denoted by $\delta_{P}$ or $\delta$ when there is no ambiguity. The symbol $\circ$ is explained below (Rem. 2.1). 
In order to deal with existence and uniqueness of the solution of (2.1) we consider the corresponding linear problem

$$
v(t, x)=u_{t_{0}}(x)+i \int_{t_{0}}^{t} P\left(i \frac{\partial}{\partial x}\right) v(\theta, x) \circ \mathrm{d} W(\theta), \quad(t, x) \in\left[t_{0}, T\right] \times \mathbb{R} .
$$

Applying Fourier transform, we have

$$
\mathcal{F}(v)(t, \xi)=\mathcal{F}\left(u_{t_{0}}\right)(x)+i \int_{t_{0}}^{t} P(\xi) \mathcal{F}(v)(\theta, \xi) \circ \mathrm{d} W(\theta), \quad(t, \xi) \in\left[t_{0}, T\right] \times \mathbb{R},
$$

then we construct the unique solution of $(2.2)$ as $v:(t, x) \rightarrow X\left(t_{0}, t\right) u_{t_{0}}(x)$ where

$$
X\left(t_{0}, t\right) u_{t_{0}}(x)=\mathcal{F}^{-1}\left(\xi \rightarrow \mathrm{e}^{i P(\xi)\left(W(t)-W\left(t_{0}\right)\right)} \mathcal{F}\left(u_{t_{0}}\right)(\xi)\right)(x) .
$$

Remark 2.1. If $W$ is a Brownian motion, using the Itô formula we prove that the solution obtained in (2.4) is the Stratonovich solution. This is why we use the notation o in (2.2) and (2.1).

Remark 2.2. Because of the properties of Fourier transform, for every $k \in \mathbb{N}$, if $u_{t_{0}} \in H^{k}$, then

$$
\left\|X\left(t_{0}, t\right) u_{t_{0}}\right\|_{H^{k}}=\left\|u_{t_{0}}\right\|_{H^{k}} .
$$

Hence, equation (2.1) is understood as the integral equation

$$
u(t, x)=X\left(t_{0}, t\right) u_{t_{0}}(x)+\int_{t_{0}}^{t} X(\theta, t) g(u(\theta, x)) \mathrm{d} \theta, \quad(t, x) \in\left[t_{0}, T\right] \times \mathbb{R} .
$$

We have the following preliminary result.

Theorem 2.3. If $u_{t_{0}} \in L^{2}$ and $g$ is Lipschitz, then there exists a unique solution $u$ with sample paths in $\mathcal{C}\left(\left[t_{0}, T\right], L^{2}\right)$ to equation (2.6). Moreover, if there exists $k \in \mathbb{N}^{*}$ such that $u_{t_{0}} \in H^{k}$ and $g \in \mathcal{B}_{k}$, then there exists a (deterministic) constant $C_{\infty, k}>0$, independent of $W$, such that

$$
\max _{t \in\left[t_{0}, T\right]}\|u(t, \cdot)\|_{H^{k}} \leq C_{\infty, k}<\infty \text { and } \max _{t \in\left[t_{0}, T\right]}\|g(u(t, \cdot))\|_{H^{k}} \leq C_{\infty, k}<\infty
$$

The constant $C_{\infty, k}$ depends on $\left\|u_{t_{0}}\right\|_{H^{k}}$ and $g$.

The proof is postponed to Section 4 . We define the family of operators $\left\{S\left(t_{0}, t\right)\right\}_{t \in\left[t_{0}, T\right]}$ such that $(t, x) \mapsto$ $S\left(t_{0}, t\right) u_{t_{0}}(x)$ is the unique solution to equation (2.6).

\subsection{Time-splitting scheme}

From now on we assume that $T=1$, we fix an initial condition $u_{0} \in L^{2}$ and $u$ denotes the solution $u$ : $(t, x) \mapsto S(0, t) u_{0}(x)$ to equation $(2.6)$ when $t_{0}=0$ and with the initial condition $u_{0}$. This subsection is devoted to introduce a splitting scheme to approximate $u$.

For $t_{0} \in[0,1]$ and $u_{t_{0}} \in L^{2}$, we introduce the problem

$$
w(t, x)=u_{t_{0}}(x)+\int_{t_{0}}^{t} g(w(\theta, x)) \mathrm{d} \theta, \quad(t, x) \in\left[t_{0}, 1\right] \times \mathbb{R} .
$$

If $g$ is a Lipschitz function, the unique solution $w$ of (2.7) is given by Theorem 2.3 (with $W \equiv 0$ ). We then define the family of operators $Y=\{Y(t)\}_{t \geq 0}$ such that for every $(t, x) \in\left[t_{0}, 1\right] \times \mathbb{R}, w(t, x)=Y\left(t-t_{0}\right) u_{t_{0}}$. We define the (Lie) splitting operator by

$$
Z\left(t_{0}, t\right):=Y\left(t-t_{0}\right) X\left(t_{0}, t\right)
$$


Let $N \in \mathbb{N}^{*}$ and $h=1 / N$. For every $k \in \mathbb{N}^{*}$ such that $k \leq 1 / h=N$, we set $S^{k, h}:=S((k-1) h, k h)$ and $Z^{k, h}:=Z((k-1) h, k h)$. For every $n \in \mathbb{N}^{*}$ such that $n \leq 1 / h=N$, we set

$$
u^{n, h}:=Z^{n, h} \ldots Z^{1, h} u_{0} .
$$

We aim to prove that $\left\{u^{n, h}\right\}_{n \in\{1, \ldots, N\}}$ approximates $\{u(n h, \cdot)\}_{n \in\{1, \ldots, N\}}$ in some sense for $h \rightarrow 0$.

For every $0 \leq t_{0}<t \leq 1$ we define

$$
\mathcal{I}_{W}\left(t_{0}, t\right):=\int_{t_{0}}^{t}\left(|W(t)-W(\theta)|+\int_{t_{0}}^{\theta}|W(\theta)-W(\sigma)| \mathrm{d} \sigma\right) \mathrm{d} \theta .
$$

Our main result is the following theorem. The proof is given in Section 4 .

Theorem 2.4. We assume that $u_{0} \in H^{\delta}, W$ admits finite first-order moments and $g \in \mathcal{B}_{\delta}$. There exists a constant $C$ which depends only on $g$ and $\left\|u_{0}\right\|_{H^{\delta}}$, such that for all $N \in \mathbb{N}^{*}$,

$$
\mathbb{E}\left[\max _{n \in\{1, \ldots, N\}}\left\|u^{n, h}-u(n h, \cdot)\right\|_{L^{2}}\right] \leq C \sum_{n=1}^{N} \mathbb{E}\left[\mathcal{I}_{W}((n-1) h, n h)\right] .
$$

Remark 2.5 (Fundamental remark). Notice that the constant $C$ appearing in Theorem 2.4 is independent of the process $W$. This is a key point of the result.

We can easily deduce the following corollary about processes with stationary increments. The proof can be omitted.

Corollary 2.6. Under the assumptions of Theorem 2.4, if $W$ has stationary increments, then (2.10) can be written as

$$
\mathbb{E}\left[\max _{n \in\{1, \ldots, N\}}\left\|u^{n, h}-u(n h, \cdot)\right\|_{L^{2}}\right] \leq C N \int_{0}^{h} \mathbb{E}[|W(\theta)|] \mathrm{d} \theta .
$$

We refer the reader to Section 3 for applications of Corollary 2.6 to equations driven by Brownian motions and (multi-)fractional processes.

Remark 2.7. Notice that existence and uniqueness of global solutions for stochastic Schrödinger equations are studied in $[11,12]$ in the cases of cubic and quintic nonlinearity. Moreover, the convergence of a splitting scheme for local solutions are established in [27] in the case of cubic nonlinearity. Nevertheless, for the sake of simplicity and because the greatest generality on the nonlinear term is not our aim in this paper, we have chosen to work under strong boundedness assumptions on the function $g$. In particular, we can see in the proofs that the constants appearing in the uniform estimates depend on upper bounds of some derivatives of $g$.

\subsection{Asymptotic-preserving property}

This subsection is devoted to establish the so-called asymptotic-preserving property of the Lie scheme for the family of equations above. From now on, the process $W$ driving equation (2.1) may vary. We then introduce new notations to take account of the dependence of all quantities with respect to the process. For a given stochastic process $W$ whose sample paths are continuous on $[0,1]$ and $\left(t_{0}, t\right) \in[0,1]^{2}$ satisfying $t_{0}<t$, we define the operators $S_{W}\left(t_{0}, t\right), X_{W}\left(t_{0}, t\right)$ and $Y_{W}\left(t_{0}, t\right)$ such that for all $u_{t_{0}} \in L^{2}$ the functions $S_{W}\left(t_{0}, \cdot\right) u_{t_{0}}, X_{W}\left(t_{0}, \cdot\right) u_{t_{0}}$ and $Y_{W}\left(t_{0}, \cdot\right) u_{t_{0}}$ are respectively solutions of $(2.1),(2.2)$ and $(2.7)$. We let $Z_{W}\left(t_{0}, t\right)=Y_{W}\left(t_{0}, t\right) X_{W}\left(t_{0}, t\right)$. For all $N \in \mathbb{N}^{*}$ and $k \in\{1, \ldots, N\}$, denoting $h:=1 / N$, we define $S_{W}^{k, h}:=S_{W}((k-1) h, k h)$ and $Z_{W}^{k, h}:=$ $Z_{W}((k-1) h, k h)$. For every $n \in\{1, \ldots, N\}$ and an initial condition $u_{0} \in H^{\delta}$, we set $u_{W}^{n, h}:=Z_{W}^{n, h} \ldots Z_{W}^{1, h} u_{0}$. We denote by $u_{W}$ the solution of equation (2.1) with $t_{0}=0$ and driven by $W$ and we set $u_{W}^{\cdot, h}:=\left\{u_{W}^{n, h}\right\}_{n \in\{1, \ldots, N\}}$. 
We consider a family of continuous processes $\left\{W^{\varepsilon}\right\}_{\varepsilon>0}$ and another continuous process $W^{0}$ such that the solution $u_{W^{\varepsilon}}$ converges to $u_{W^{0}}$ as $\varepsilon \rightarrow 0$. We establish and prove in other sections that the schemes $u_{W^{\varepsilon}}^{, h}$ and $u_{W^{0}}^{, h}$ converge respectively to $W^{\varepsilon}$ and $W^{0}$ as the time step $h$ goes to 0 . Under suitable assumptions on the sequence of processes $\left\{W^{\varepsilon}\right\}_{\varepsilon>0}$ we establish in this section that:

- The scheme $u_{W^{\varepsilon}}^{, h}$ converges (in some sense) to $u_{W^{\varepsilon}}$ uniformly with respect to $\varepsilon>0$ when $h \rightarrow 0$,

- and the scheme $u_{W^{\varepsilon}}^{, h}$ converges (in some sense) to $u_{W^{0}}^{, h}$ as $\varepsilon \rightarrow 0$ for every $h>0$.

This implies that the limit $\varepsilon \rightarrow 0$ does not affect the convergence of the splitting scheme. This is the so-called Asymptotic-Preserving (AP) property, which is usually represented by the diagram

$$
\begin{gathered}
u_{W^{\varepsilon} \rightarrow 0}^{, h} \stackrel{\varepsilon \rightarrow 0}{\longrightarrow} u_{W^{0}}^{, h} \\
\downarrow^{h \rightarrow 0} \\
u_{W^{\varepsilon}} \stackrel{\varepsilon \rightarrow 0}{\longrightarrow} u_{W^{0}}
\end{gathered}
$$

We establish the main results of this subsection. The proofs are given in Section 4. From now on, we suppose that $\left\{W^{\varepsilon}\right\}_{\varepsilon>0}$ and $W^{0}$ satisfy the following assumptions.

- Assumption (A1). As $\varepsilon \rightarrow 0, W^{\varepsilon}$ converges in distribution to $W^{0}$ in the space $\mathcal{C}([0,1], \mathbb{R})$.

- Assumption (A2). There exist three constants $K>0, \gamma \geq 1$ and $\beta \geq 1$ such that for all $t_{1}$ and $t_{2} \in[0,1]$ and $\varepsilon>0$,

$$
\mathbb{E}\left[\left(W^{\varepsilon}\left(t_{1}\right)-W^{\varepsilon}\left(t_{2}\right)\right)^{\gamma}\right] \leq K\left|t_{1}-t_{2}\right|^{\beta}
$$

The first result concerns the convergence of $u_{W^{\varepsilon}}$ when $\varepsilon \rightarrow 0$.

Theorem 2.8. Let $u_{0} \in H^{\delta}$. The mapping

$$
\begin{aligned}
\mathcal{C}([0,1], \mathbb{R}) & \rightarrow \mathcal{C}\left([0,1], L^{2}\right) \\
w & \longmapsto u_{w}
\end{aligned}
$$

is Lipschitz. As a consequence, when $\varepsilon \rightarrow 0, u_{W^{\varepsilon}}$ converges in distribution to $u_{W^{0}}$ in $\mathcal{C}\left([0,1], L^{2}\right)$.

The second main result of this section deals with the convergence of $u_{W^{\varepsilon}}^{\prime, h}$ as $\varepsilon \rightarrow 0$.

Theorem 2.9. Let $u_{0} \in H^{\delta}, N \in \mathbb{N}^{*}$ and $h=1 / N$. The mapping

$$
\begin{aligned}
\mathcal{C}([0,1], \mathbb{R}) & \rightarrow\left(L^{2}\right)^{N+1} \\
w & \longmapsto\left\{u_{w}^{j, h}\right\}_{j=0, \ldots, N}
\end{aligned}
$$

is Lipschitz. As a consequence, when $\varepsilon \rightarrow 0,\left\{u_{W^{\varepsilon}}^{j, h}\right\}_{j=0, \ldots, N}$ converges in distribution to $\left\{u_{W^{0}}^{j, h}\right\}_{j=0, \ldots, N}$ in $\left(L^{2}\right)^{N+1}$ as $\varepsilon \rightarrow 0$.

We state the last main result of this section.

Theorem 2.10. We assume that $u_{0} \in H^{\delta}$ and $g \in \mathcal{B}_{\delta}$. Then there exists a constant $C$ which depends only on $g$ and $\left\|u_{0}\right\|_{H^{\delta}}$, such that for every $h \in(0,1]$ and every $\varepsilon \geq 0$,

$$
\mathbb{E}\left[\max _{n \in\{1, \ldots, N\}}\left\|u_{W^{\varepsilon}}^{n, h}-u_{W^{\varepsilon}}(n h, \cdot)\right\|_{L^{2}}\right] \leq C h^{\beta / \gamma} .
$$

This implies the AP property described by diagram (2.12). 


\section{Applications}

This section is devoted to apply Theorem 2.10 to different frameworks. From now on, we assume that $u_{0} \in H^{\delta}$ and $g \in \mathcal{B}_{\delta}$.

\subsection{Diffusion approximation}

In this subsection we improve results from [27]. For all $\varepsilon>0$, consider the solution $u^{\varepsilon}:[0,1] \times \mathbb{R} \rightarrow \mathbb{C}$ of the equation

$$
\left\{\begin{array}{l}
\frac{\partial u^{\varepsilon}}{\partial t}(t, x)=\frac{i}{\varepsilon} m\left(\frac{t}{\varepsilon^{2}}\right) P\left(i \frac{\partial}{\partial x}\right) u^{\varepsilon}(t, x)+g\left(u^{\varepsilon}(t, x)\right), \\
u^{\varepsilon}(t=0, x)=u_{0}(x),
\end{array} \text { for all }(t, x) \in[0,1] \times \mathbb{R},\right.
$$

where $m$ is a continuous, centered, stationary and mixing process [18] with finite second order moments. Let $B$ be a Brownian motion and $c_{0}$ be a positive constant defined by

$$
c_{0}^{2}=2 \int_{0}^{\infty} \mathbb{E}[m(0) m(\theta)] \mathrm{d} \theta
$$

For every $t \geq 0$ we set

$$
\mathcal{S}^{\varepsilon}(t)=\frac{1}{\varepsilon} \int_{0}^{t} m\left(\frac{\theta}{\varepsilon^{2}}\right) \mathrm{d} \theta
$$

It well-known that $\left\{\mathcal{S}^{\varepsilon}(t)\right\}_{t \in[0,1]}$ converges in distribution to $c_{0} B$ in $\mathcal{C}([0,1])$ as $\varepsilon \rightarrow 0$ (the functional Donsker theorem, see [18] for instance). As a consequence, by Theorem 2.8, $u^{\varepsilon}$ converges in distribution to $u$ in the space $\mathcal{C}\left([0,1], L^{2}\right)$ as $\varepsilon \rightarrow 0$, where $u$ is the solution of

$$
u(t, x)=u_{0}(x)+i c_{0} \int_{0}^{t} P\left(i \frac{\partial}{\partial x}\right) u(\theta, x) \circ d B(\theta)+\int_{0}^{t} g(u(\theta, x)) \mathrm{d} \theta, \text { for all }(t, x) \in[0,1] \times \mathbb{R} .
$$

For every $N \in \mathbb{N}^{*}$ (with $h=1 / N$ ), let $\left\{u^{n, h, \varepsilon}\right\}_{0 \leq n \leq N}$ be the Lie scheme associated to $u^{\varepsilon}$ and $\left\{u^{n, h}\right\}_{0 \leq n \leq N}$ be the Lie scheme associated to $u$. We now establish the AP property in this framework.

Theorem 3.1. For all $N \in \mathbb{N}^{*},\left\{u^{n, h, \varepsilon}\right\}_{0 \leq n \leq N}$ converges in distribution to $\left\{u^{n, h}\right\}_{0 \leq n \leq N}$ in $\left(L^{2}\right)^{N+1}$ as $\varepsilon \rightarrow 0$. Moreover, there exists a constant $C$ which depends only on $g$ and $\left\|u_{0}\right\|_{H^{\delta}}$, such that for every $h \in(0,1]$ and every $\varepsilon>0$,

$$
\mathbb{E}\left[\max _{n \in\{1, \ldots, N\}}\left\|u^{n, h, \varepsilon}-u^{\varepsilon}(n h, \cdot)\right\|_{L^{2}}\right] \leq C h^{1 / 2} \text { and } \mathbb{E}\left[\max _{n \in\{1, \ldots, N\}}\left\|u^{n, h}-u(n h, \cdot)\right\|_{L^{2}}\right] \leq C h^{1 / 2} .
$$

A weaker form of this result has been proven in [27]. More precisely, it has been shown that, for $P(\xi)=\xi^{2}$, there exists a constant $C$ which depends only on $g$ and $\left\|u_{0}\right\|_{H^{2}}$ (because $\delta=2$ in this case), such that for every $h \in(0,1]$ and every $\varepsilon>0$,

$$
\max _{n \in\{1, \ldots, N\}} \mathbb{E}\left[\left\|u^{n, h, \varepsilon}-u^{\varepsilon}(n h, \cdot)\right\|_{L^{2}}\right] \leq C\left(h^{1 / 2}+\varepsilon\right) .
$$

The improvement comes from the general formulation of the error estimate in Theorem 2.4 and in particular from the use of a modified Gronwall lemma in its proof. 
Proof. The convergence of $\left\{u^{n, h, \varepsilon}\right\}_{0 \leq n \leq N}$ to $\left\{u^{n, h}\right\}_{0 \leq n \leq N}$ is a direct consequence of Theorem 2.9 and the functional Donsker theorem. To prove (3.3), we show Assumptions (A1) and (A2). For all $s<t \in[0,1]$, because $m$ is stationary, we have

$$
\begin{aligned}
\mathbb{E}\left[\left(\mathcal{S}^{\varepsilon}(t)-\mathcal{S}^{\varepsilon}(s)\right)^{2}\right] & \leq \frac{1}{\varepsilon^{2}} \int_{s}^{t} \mathrm{~d} \theta \int_{s}^{t} \mathrm{~d} \sigma\left|\mathbb{E}\left[m\left(\frac{\theta}{\varepsilon^{2}}\right) m\left(\frac{\sigma}{\varepsilon^{2}}\right)\right]\right|=\frac{1}{\varepsilon^{2}} \int_{s}^{t} \mathrm{~d} \theta \int_{s}^{t} \mathrm{~d} \sigma\left|\mathbb{E}\left[m\left(\frac{\theta-\sigma}{\varepsilon^{2}}\right) m(0)\right]\right| \\
& \leq \frac{2}{\varepsilon^{2}} \int_{s}^{t} \mathrm{~d} \theta \int_{0}^{\theta} \mathrm{d} \sigma\left|\mathbb{E}\left[m\left(\frac{\sigma}{\varepsilon^{2}}\right) m(0)\right]\right|=2 \int_{s}^{t} \mathrm{~d} \theta \int_{0}^{\theta / \varepsilon^{2}} \mathrm{~d} \sigma|\mathbb{E}[m(\sigma) m(0)]| \\
& \leq 2(t-s) \int_{0}^{\infty} \mathrm{d} \sigma|\mathbb{E}[m(\sigma) m(0)]| .
\end{aligned}
$$

Hence, Assumptions (A1) and (A2) are satisfied. This ends the proof.

\subsection{Approximation by a fractional Brownian motion}

A fractional Brownian motion $B_{H}=\left\{B_{H}(t)\right\}_{t \geq 0}$ (see [31]) with Hurst index $H \in(0,1)$ is a Gaussian process with mean 0 and satisfying for all $t$ and $s \geq 0$,

$$
\mathbb{E}\left[B_{H}(t) B_{H}(s)\right]=\frac{1}{2}\left(t^{2 H}+s^{2 H}-|t-s|^{2 H}\right) .
$$

Notice that a fractional Brownian motion with $H=1 / 2$ is a Brownian motion.

The class of fractional Brownian motions is important in applications of stochastic processes because it satisfies the invariance principle stated below.

Let $H \in(0,1)$ and $m$ be a stationary Gaussian process with mean 0 . For every $\varepsilon \in(0,1)$ we define $\mathcal{S}^{\varepsilon}=$ $\left\{\mathcal{S}^{\varepsilon}(t)\right\}_{t \geq 0}$ such that for every $t \geq 0$,

$$
\mathcal{S}^{\varepsilon}(t)=\varepsilon^{2 H} \int_{0}^{t / \varepsilon^{2}} m(s) \mathrm{d} s .
$$

We assume that one of the three following properties holds:

- If $H \in(1 / 2,1)$, there exists $\sigma_{H}>0$ such that $\mathbb{E}[m(0) m(t)] \sim \sigma_{H} t^{2 H-2}$ as $t \rightarrow \infty$.

- If $H \in(0,1 / 2)$, there exists $\sigma_{H}<0$ such that $\mathbb{E}[m(0) m(t)] \sim \sigma_{H} t^{2 H-2}$ as $t \rightarrow \infty$ and $\int_{0}^{\infty} \mathbb{E}[m(0) m(t)] \mathrm{d} t=0$.

- If $H=1 / 2, \int_{0}^{\infty}|\mathbb{E}[m(0) m(t)]| \mathrm{d} t<\infty$ and $\int_{0}^{\infty} \mathbb{E}[m(0) m(t)] \mathrm{d} t>0$.

We have the following result (invariance principle, see [31]).

Lemma 3.2. As $\varepsilon \rightarrow 0, \mathcal{S}^{\varepsilon}$ converges in distribution in $\mathcal{C}([0, \infty))$ to $c_{H} B_{H}$ where $B_{H}$ is a fractional Brownian motion with Hurst index $H$ and $c_{H}$ is a positive constant defined by $c_{H}^{2}=2 \int_{0}^{\infty} \mathbb{E}[m(0) m(t)] \mathrm{d} t$ if $H=1 / 2$ and by $c_{H}^{2}=\sigma_{H} / H(2 H-1)$ if $H \neq 1 / 2$.

For all $\varepsilon>0$, consider the solution $u^{\varepsilon}:[0,1] \times \mathbb{R} \rightarrow \mathbb{C}$ of the equation

$$
\left\{\begin{array}{l}
\frac{\partial u^{\varepsilon}}{\partial t}(t, x)=\frac{i}{\varepsilon^{2-2 H}} m\left(\frac{t}{\varepsilon^{2}}\right) P\left(i \frac{\partial}{\partial x}\right) u^{\varepsilon}(t, x)+g\left(u^{\varepsilon}(t, x)\right), \\
u^{\varepsilon}(t=0, x)=u_{0}(x),
\end{array} \text { for all }(t, x) \in[0,1] \times \mathbb{R},\right.
$$

where $m$ is defined just above. Thanks to Lemmas 3.2 and $2.8, u^{\varepsilon}$ converges in distribution to $u:[0,1] \times \mathbb{R} \rightarrow \mathbb{C}$ in the space $\mathcal{C}\left([0,1], L^{2}\right)$ as $\varepsilon \rightarrow 0$, where $u$ is the solution of

$$
u(t, x)=u_{0}(x)+i c_{H} \int_{0}^{t} P\left(i \frac{\partial}{\partial x}\right) u(\theta, x) \circ d B_{H}(\theta)+\int_{0}^{t} g(u(\theta, x)) \mathrm{d} \theta, \text { for all }(t, x) \in[0,1] \times \mathbb{R} .
$$

In the equation above, $c_{H}$ is the constant defined in Lemma 3.2, $B_{H}$ is a fractional Brownian motion with Hurst index $H$. For every $N \in \mathbb{N}$ (with $h=1 / N$ ), let $\left\{u^{n, h, \varepsilon}\right\}_{1 \leq n \leq N}$ be the Lie scheme associated to $u^{\varepsilon}$ and $\left\{u^{n, h}\right\}_{1 \leq n \leq N}$ be the Lie scheme associated to $u$. We establish the AP property. 
Theorem 3.3. For every $N \in \mathbb{N}^{*},\left\{u^{n, h, \varepsilon}\right\}_{1 \leq n \leq N}$ converges in distribution to $\left\{u^{n, h}\right\}_{1 \leq n \leq N}$ as $\varepsilon \rightarrow 0$. Moreover, there exists a constant $C$ which depends only on $g$ and $\left\|u_{0}\right\|_{H^{\delta}}$, such that for every $h \in(0,1]$ and every $\varepsilon>0$,

$$
\mathbb{E}\left[\max _{n \in\{1, \ldots, N\}}\left\|u^{n, h, \varepsilon}-u^{\varepsilon}(n h, \cdot)\right\|_{L^{2}}\right] \leq C h^{H}, \text { and } \mathbb{E}\left[\max _{n \in\{1, \ldots, N\}}\left\|u^{n, h}-u(n h, \cdot)\right\|_{L^{2}}\right] \leq C h^{H} .
$$

Proof. By Lemma 3.2 and Theorem 2.9, we get the convergence of $\left\{u^{n, h, \varepsilon}\right\}_{1 \leq n \leq N}$ as $\varepsilon \rightarrow 0$. By Lemma 3.2 again, $\mathcal{S}^{\varepsilon}$ satisfies Assumptions (A1). Moreover, because $m$ is Gaussian, for every $q \in \mathbb{N}^{*}$, there exists $C_{q}>0$ such that for all $t$ and $s$,

$$
\mathbb{E}\left[\left(\mathcal{S}^{\varepsilon}(t)-\mathcal{S}^{\varepsilon}(s)\right)^{2 q}\right] \leq C|t-s|^{2 H q} .
$$

Then, $\mathcal{S}^{\varepsilon}$ satisfies assumptions (A1) and (A2), which concludes the proof by Theorem 2.10.

Notice that, if $H=1 / 2$, then Theorem 3.3 is Theorem 3.1 in the Brownian case.

\subsection{Approximation in a long-range random medium}

Recently, long-range random media have attracted a lot of attention in applications to wave propagation (see $[20,29]$ for instance). A fractional Brownian motion with Hurst index $H>1 / 2$ is a basic model for longrange dependence. Nevertheless, this model is Gaussian and we also need non-Gaussian models. A natural generalization of fractional Brownian motions is the class of Hermite processes. Let $K \in \mathbb{N}^{*}$ and $H=(2-$ $\gamma K) / 2 \in(1 / 2,1)$. We define the $K$ th Hermite process of index $H$ for every $t>0$ by

$$
B_{H, K}(t)=\int_{\mathbb{R}^{K}} \mathcal{G}_{H, K}\left(t, x_{1}, \ldots, x_{K}\right) \prod_{k=1}^{K} \tilde{B}\left(\mathrm{~d} x_{k}\right)
$$

with

$$
\mathcal{G}_{H, K}\left(t, x_{1}, \ldots, x_{K}\right)=\frac{\left(\mathrm{e}^{-i t \sum_{j=1}^{K} x_{j}}-1\right)}{C(H) \sum_{j=1}^{K} x_{j}} \prod_{k=1}^{K} \frac{x_{k}}{\left|x_{k}\right|^{(H-1) / K+3 / 2}}
$$

where $C(H)$ a normalizing constant, $\tilde{B}(\mathrm{~d} x)$ is the Fourier transform of a Brownian measure and the multiple stochastic integral is in the sense of [14].

Notice that for $K=1, B_{H, K}=B_{H, 1}$ is a fractional Brownian motion with Hurst index $H>1 / 2$. More generally, for every $K, B_{H, K}$ is centered and admits the same covariance as a fractional Brownian motion, that is, for all $t$ and $s \geq 0$,

$$
\mathbb{E}\left[B_{H, K}(t) B_{H, K}(s)\right]=\frac{1}{2}\left(t^{2 H}+s^{2 H}-|t-s|^{2 H}\right) .
$$

Moreover, $B_{H, K}$ is Gaussian if and only if $K=1$.

As the class of fractional Brownian motions, Hermite processes are important in applications of stochastic processes because they satisfy the invariance principle $[15,33,34]$. Let $m$ be a continuous Gaussian process, centered, stationary, satisfying $\mathbb{E}\left[m(0)^{2}\right]=1$ and such that

$$
\mathbb{E}[m(0) m(t)] \sim c_{m} t^{-\gamma}
$$

as $t \rightarrow \infty$, where $1<\gamma<1 / K$ and $c_{m}>0$. For every $\varepsilon \in(0,1)$ we define $\mathcal{S}^{\varepsilon}=\left\{\mathcal{S}^{\varepsilon}(t)\right\}_{t \geq 0}$ for every $t \geq 0$ by

$$
\mathcal{S}^{\varepsilon}(t)=\varepsilon^{-\gamma K} \int_{0}^{t} \Phi\left(m\left(\frac{s}{\varepsilon^{2}}\right)\right) \mathrm{d} s
$$


where $\Phi$ is a continuous function in $L^{2}\left(\mathrm{e}^{-x^{2} / 2} \mathrm{~d} x\right)$ with Hermite index equal to $K \in \mathbb{N}^{*}$. This means that if we denote the $k$ th Hermite coefficient of $\Phi$ by

$$
\Phi_{k}=\int_{-\infty}^{\infty} P_{k}(x) \Phi(x) \frac{\mathrm{e}^{-x^{2} / 2}}{k ! \sqrt{2 \pi}} \mathrm{d} x
$$

where $P_{k}$ is the $k$ th Hermite polynomial, then we have

$$
\Phi=\sum_{k=K}^{\infty} \Phi_{k} P_{k}
$$

with $\Phi_{K} \neq 0$. The invariance principle for Hermite processes can be stated as below.

Lemma 3.4. As $\varepsilon \rightarrow 0, \mathcal{S}^{\varepsilon}$ converges in distribution to $c_{H, K} B_{H, K}$ in $\mathcal{C}([0, \infty))$, where $c_{H, K}^{2}=c_{m}^{K} \Phi_{K}^{2} /(K !)^{2}$.

For every $\varepsilon>0$, we consider the solution $u^{\varepsilon}$ of the equation

$$
\begin{cases}\frac{\partial u^{\varepsilon}}{\partial t}(t, x)=\frac{i}{\varepsilon^{\gamma K}} m\left(\frac{t}{\varepsilon^{2}}\right) P\left(i \frac{\partial}{\partial x}\right) u^{\varepsilon}(t, x)+g\left(u^{\varepsilon}(t, x)\right), & \text { for all }(t, x) \in[0,1] \times \mathbb{R}, \\ u^{\varepsilon}(t=0, x)=u_{0}(x), & \end{cases}
$$

where $m$ is defined just above. Thanks to Lemmas 3.4 and $2.8, u^{\varepsilon}$ converges in distribution to $u:[0,1] \times \mathbb{R} \rightarrow \mathbb{C}$ in the space $\mathcal{C}\left([0,1], L^{2}\right)$ as $\varepsilon \rightarrow 0$, where $u$ is the solution of

$$
u(t, x)=u_{0}(x)+i c_{H, K} \int_{0}^{t} P\left(i \frac{\partial}{\partial x}\right) u(\theta, x) \circ d B_{H, K}(\theta)+\int_{0}^{t} g(u(\theta, x)) \mathrm{d} \theta, \text { for all }(t, x) \in[0,1] \times \mathbb{R},
$$

with the constant $c_{H, K}$ defined as in Lemma $3.4, B_{H, K}$ is a $K$ th Hermite process of index $H$. For every $N \in \mathbb{N}^{*}$ (with $h=1 / N$ ), let $\left\{u^{n, h, \varepsilon}\right\}_{1 \leq n \leq N}$ be the Lie scheme associated to $u^{\varepsilon}$ and $\left\{u^{n, h}\right\}_{1 \leq n \leq N}$ be the Lie scheme associated to $u$. We now establish the AP property.

Theorem 3.5. For every $N \in \mathbb{N}^{*},\left\{u^{n, h, \varepsilon}\right\}_{1 \leq n \leq N}$ converges in distribution to $\left\{u^{n, h}\right\}_{1 \leq n \leq N}$ as $\varepsilon \rightarrow 0$. Moreover, there exists a constant $C$ which depends only on $g$ and $\left\|u_{0}\right\|_{H^{\delta}}$, such that for every $h \in(0,1]$ and every $\varepsilon>0$,

$$
\mathbb{E}\left[\max _{n \in\{1, \ldots, N\}}\left\|u^{n, h, \varepsilon}-u^{\varepsilon}(n h, \cdot)\right\|_{L^{2}}\right] \leq C h^{H}, \text { and } \mathbb{E}\left[\max _{n \in\{1, \ldots, N\}}\left\|u^{n, h}-u(n h, \cdot)\right\|_{L^{2}}\right] \leq C h^{H} .
$$

Proof. The proof is similar to the proof of Theorem 3.3. It is a consequence of Lemma 3.4 and Theorems 2.9 and 2.10 .

\subsection{Generalization to multifractional media}

Fractional Brownian motions with Hurst index $H>1 / 2$ and Hermite processes fit very well for modeling long-range media. Nevertheless, their range properties are governed by the constant Hurst index, which implies a strong homogeneity. To deal with less homogeneous media, multifractional processes have been introduced $[5,30]$. The main interest of multifractional processes lies in the fact that they have a Hurst index varying along their trajectories. This implies more flexibility in the choice of the models. Applications of multifractional models to waves in random media have been studied recently [29]. In this subsection we deal with the AP property of the Lie time-splitting scheme in the case of convergence to multifractional processes. We restrict our study to a simple Gaussian framework, but it can be easily generalized to non-Gaussian settings as discussed at the end of this subsection. 
Let $m=\{m(t, H)\}_{(t, H) \in \mathbb{R} \times(1 / 2,1)}$ be a Gaussian field. We assume that $m$ is centered and satisfies for every compact set $K \subset(1 / 2,1)$,

$$
\lim _{\left|t_{1}-t_{2}\right| \rightarrow \infty} \sup _{\left(H_{1}, H_{2}\right) \in K^{2}}\left|R\left(H_{1}, H_{2}\right)-\right| t_{1}-\left.t_{2}\right|^{2-H_{1}-H_{2}} \mathbb{E}\left[m\left(t_{1}, H_{1}\right) m\left(t_{2}, H_{2}\right)\right] \mid=0,
$$

where $R:(1 / 2,1)^{2} \rightarrow(0, \infty)$ is a continuous function. This is long-range assumption in a multifractional setting. Let $\mathcal{H}:[0, \infty) \rightarrow[a, b] \subset(1 / 2,1)$ be a continuous function. We define $\mathcal{S}^{\varepsilon}=\left\{\mathcal{S}^{\varepsilon}(t)\right\}_{t \geq 0}$ such that for every $t \geq 0$,

$$
\mathcal{S}^{\varepsilon}(t)=\int_{0}^{t / \varepsilon^{2}} \varepsilon^{2 \mathcal{H}\left(\varepsilon^{2} s\right)} m\left(s, \mathcal{H}\left(\varepsilon^{2} s\right)\right) \mathrm{d} s=\int_{0}^{t} \varepsilon^{2 \mathcal{H}(s)-2} m\left(s / \varepsilon^{2}, \mathcal{H}(s)\right) \mathrm{d} s .
$$

The following result establishes an invariance principle for Gaussian multifractional processes ([9], see [28, 29] for generalizations and applications).

Lemma 3.6. As $\varepsilon \rightarrow 0, \mathcal{S}^{\varepsilon}$ converges in distribution to a process $\mathcal{S}_{\mathcal{H}}$ in $\mathcal{C}([0, \infty))$, where $\mathcal{S}_{\mathcal{H}}$ is Gaussian, centered and satisfies for all $t$ and $s \geq 0$,

$$
\mathbb{E}\left[\mathcal{S}_{\mathcal{H}}(t) \mathcal{S}_{\mathcal{H}}(s)\right]=\int_{0}^{t} \mathrm{~d} \theta \int_{0}^{s} \mathrm{~d} \sigma R(\mathcal{H}(\theta), \mathcal{H}(\sigma))|\theta-\sigma|^{\mathcal{H}(\theta)+\mathcal{H}(\sigma)-2} .
$$

A detailed study of $\mathcal{S}_{\mathcal{H}}$ can be found in [9]. In particular, it is proven that $S_{\mathcal{H}}$ satisfies the main properties of a multifractional process.

For every $\varepsilon>0$, we consider the solution $u^{\varepsilon}:[0,1] \times \mathbb{R} \rightarrow \mathbb{C}$ of the equation

$$
\left\{\begin{array}{l}
\frac{\partial u^{\varepsilon}}{\partial t}(t, x)=\frac{i}{\varepsilon^{2-2 \mathcal{H}(s)}} m\left(\frac{s}{\varepsilon^{2}}, \mathcal{H}(s)\right) P\left(i \frac{\partial}{\partial x}\right) u^{\varepsilon}(t, x)+g\left(u^{\varepsilon}(t, x)\right), \\
u^{\varepsilon}(t=0, x)=u_{0}(x),
\end{array} \text { for all }(t, x) \in[0,1] \times \mathbb{R},\right.
$$

where $m$ is defined just above. Because of Lemmas 3.6 and $2.8, u^{\varepsilon}$ converges in distribution to $u:[0,1] \times \mathbb{R} \rightarrow \mathbb{C}$ in the space $\mathcal{C}\left([0,1], L^{2}\right)$ as $\varepsilon \rightarrow 0$, where $u$ is the solution of

$$
u(t, x)=u_{0}(x)+i \int_{0}^{t} P\left(i \frac{\partial}{\partial x}\right) u(\theta, x) \circ \mathrm{d} \mathcal{S}_{\mathcal{H}}(\theta)+\int_{0}^{t} g(u(\theta, x)) \mathrm{d} \theta, \text { for all }(t, x) \in[0,1] \times \mathbb{R} .
$$

In the equation above, $\mathcal{S}_{\mathcal{H}}$ is the multifractional process defined in Lemma 3.6. For every $N \in \mathbb{N}^{*}$ (with $h=1 / N$ ), let $\left\{u^{n, h, \varepsilon}\right\}_{1 \leq n \leq N}$ be the Lie scheme associated to $u^{\varepsilon}$ and $\left\{u^{n, h}\right\}_{1 \leq n \leq N}$ be the Lie scheme associated to $u$. We have the following result.

Theorem 3.7. For every $N \in \mathbb{N}^{*},\left\{u^{n, h, \varepsilon}\right\}_{1 \leq n \leq N}$ converges in distribution to $\left\{u^{n, h}\right\}_{1 \leq n \leq N}$ as $\varepsilon \rightarrow 0$. Moreover, there exists a constant $C$ which depends only on $g$ and $\left\|u_{0}\right\|_{H^{\delta}}$, such that for every $h \in(0,1]$ and every $\varepsilon>0$,

$$
\mathbb{E}\left[\max _{n \in\{1, \ldots, N\}}\left\|u^{n, h, \varepsilon}-u^{\varepsilon}(n h, \cdot)\right\|_{L^{2}}\right] \leq C h^{\inf \mathcal{H}} \text { and } \mathbb{E}\left[\max _{n \in\{1, \ldots, N\}}\left\|u^{n, h}-u(n h, \cdot)\right\|_{L^{2}}\right] \leq C h^{\inf \mathcal{H}} .
$$

Proof. From the covariance of $S_{\mathcal{H}}$, we can easily deduce that there exists $C>0$ such that for all $t$ and $s$,

$$
\mathbb{E}\left[\left(\mathcal{S}^{\varepsilon}(t)-\mathcal{S}^{\varepsilon}(s)\right)^{2}\right] \leq C|t-s|^{2 \inf \mathcal{H}} .
$$

The remaining part of the proof is similar to the proof of Theorem 3.3.

To conclude this section, notice that we can also pursue the same study for non-Gaussian multifractional models by using Hermite processes and replacing Lemma 3.6 for suitable limit theorems (see $[28,29]$ for invariance principle for non-Gaussian multifractional processes). 


\section{Proofs}

\subsection{Proof of Theorem 2.3}

Throughout this section, we consider a continuous sample path $W$ of a given stochastic process. Notice that all the constants appearing in this proof are independent of $W$, even though the other quantities do depend on $W$. For the sake of simplicity, we assume that $t_{0}=0$ and $T=1$.

Let $\Gamma$ be the application from $\mathcal{C}\left([0,1], L^{2}\right)$ to itself such that for every $U \in \mathcal{C}\left([0,1], L^{2}\right)$,

$$
\Gamma(U)(t, x)=X(0, t) u_{0}(x)+\int_{0}^{t} X(s, t) g(U(s, \cdot))(x) d s .
$$

We define the sequence $\left\{U_{j}\right\}_{j \in \mathbb{N}} \in\left(\mathcal{C}\left([0,1], L^{2}\right)\right)^{\mathbb{N}}$ by $U_{0}:=u_{0}$ and $U_{j+1}:=\Gamma\left(U_{j}\right)$ for all $j>0$. Using a classical fixed-point procedure, we get the following result.

Lemma 4.1. There exists a unique solution $u \in \mathcal{C}\left([0,1], L^{2}\right)$ to $(2.6)$.

Proof. Obviously, for every $U$ and $V$ in $\mathcal{C}\left([0,1], L^{2}\right)$ and every $t \in[0,1]$,

$$
\sup _{t^{\prime} \in[0, t]}\left\|\Gamma\left(U\left(t^{\prime}\right)\right)-\Gamma\left(V\left(t^{\prime}\right)\right)\right\|_{L^{2}} \leq\|g\|_{L i p} \int_{0}^{t} \sup _{t^{\prime} \in[0, \theta]}\left\|U\left(t^{\prime}\right)-V\left(t^{\prime}\right)\right\|_{L^{2}} \mathrm{~d} \theta
$$

Moreover, since $g(0)=0$, we have

$$
\sup _{t^{\prime} \in[0, t]}\left\|\Gamma\left(U\left(t^{\prime}\right)\right)\right\|_{L^{2}} \leq\left\|u_{0}\right\|_{L^{2}}+\|g\|_{L i p} \int_{0}^{t} \sup _{t^{\prime} \in[0, \theta]}\left\|U\left(t^{\prime}\right)\right\|_{L^{2}} \mathrm{~d} \theta
$$

and thus

$$
\sup _{t \in[0,1]}\|\Gamma(U(t))\|_{L^{2}} \leq\left\|u_{0}\right\|_{L^{2}} \exp \left(\|g\|_{L i p}\right) .
$$

We deduce from (4.1) and (4.2) that the sequence $\left\{U_{j}\right\}_{j \in \mathbb{N}}$ is a Cauchy sequence in $\mathcal{C}\left([0,1], L^{2}\right)$. Then there exists a solution $u$ to (2.6). The uniqueness is a direct consequence of (4.1).

We now prove the estimates on the $H^{k}$-norm of the solution $u$. Let us first give the following useful lemma.

Lemma 4.2. Let $n \geq 1, \psi \in \mathcal{C}^{n}\left(\mathbb{R}, \mathbb{R}^{2}\right)$ and $\phi \in \mathcal{C}^{n}\left(\mathbb{R}^{2}, \mathbb{R}\right)$. There exists a constant $C>0$ such that for every $x \in \mathbb{R}$,

$$
\left|(\phi \circ \psi)^{(n)}(x)\right| \leq C \sum_{k=1}^{n}\left\|\nabla^{k} \phi\right\|_{\infty} \sum_{\substack{1 \leq l_{1} \leq \ldots \leq l_{k} \leq n \\ l_{1}+\ldots+l_{k}=n}} \prod_{r=1}^{k}\left\|\psi^{\left(l_{r}\right)}(x)\right\|
$$

Remark 4.3. In the previous result, remark that

$$
\sum_{k=1}^{n}\left\|\nabla^{k} \phi\right\|_{\infty} \sum_{\substack{1 \leq l_{1} \leq \ldots \leq l_{k} \leq n \\ l_{1}+\ldots+l_{k}=n}} \prod_{r=1}^{k}\left\|\psi^{\left(l_{r}\right)}(x)\right\|=\|\nabla \phi\|_{\infty}\left\|\psi^{(n)}(x)\right\|+\sum_{k=2}^{n}\left\|\nabla^{k} \phi\right\|_{\infty} \sum_{\substack{1 \leq l_{1} \leq \ldots \leq l_{k} \leq n \\ l_{1}+\ldots+l_{k}=n}} \prod_{r=1}^{k}\left\|\psi^{\left(l_{r}\right)}(x)\right\|
$$

The proof of Lemma 4.2 is a direct consequence of Lemma 4.4 stated just below and whose proof is omitted. It can be proven by induction. 
Lemma 4.4. Let $n \geq 1, \psi=\left(\psi_{1}, \psi_{2}\right) \in \mathcal{C}^{n}\left(\mathbb{R}, \mathbb{R}^{2}\right)$ and $\phi \in \mathcal{C}^{n}\left(\mathbb{R}^{2}, \mathbb{R}\right)$. Then

$$
(\phi \circ \psi)^{(n)}=\sum_{\substack{j, k \geq 0 \\ 1 \leq j+k \leq n}} \sum_{\substack{1 \leq l_{1} \leq \ldots \leq l_{k} \leq n \\ 1 \leq m_{1} \leq \ldots \leq m_{j} \leq n \\ l_{1}+\ldots+m_{1}+\ldots=n}} w_{l_{1}, \ldots, m_{1}, \ldots}\left(\phi^{(k, j)} \circ \psi\right)\left(\prod_{r=1}^{k} \psi_{1}^{\left(l_{r}\right)}\right)\left(\prod_{r=1}^{j} \psi_{2}^{\left(m_{r}\right)}\right)
$$

where all the coefficients $w_{l_{1}, \ldots, m_{1}, \ldots}$ are integer.

We now establish the following lemma.

Lemma 4.5. Let $u_{0} \in H^{n}, n \geq 1$. Then there exists a deterministic constant $C_{n}=C\left(g,\left\|u_{0}\right\|_{H^{n}}\right)$ depending only on $\left\|u_{0}\right\|_{H^{n}}$ and $g$ such that the solution $u$ of (2.6) satisfies

$$
\sup _{t \in[0,1]}\|u(t)\|_{H^{n}} \leq C_{n}
$$

The proof of Lemma 4.5 is a direct consequence of Lemma 4.6 stated just below.

Lemma 4.6. Let $u_{0} \in H^{n}, n \geq 1$. Then there exists a deterministic constant $C_{n}=C\left(g,\left\|u_{0}\right\|_{H^{n}}\right)$ depending only on $\left\|u_{0}\right\|_{H^{n}}$ and $g$ such that for every $j \geq 1, U_{j}$ satisfies

$$
\sup _{t \in[0,1]}\left\|U_{j}(t)\right\|_{H^{n}} \leq C_{n} .
$$

Proof. We prove by induction that for all $n \in \mathbb{N}$ and $j \in \mathbb{N}$,

$$
\left\|U_{j+1}(t)\right\|_{H^{n}} \leq\left\|u_{0}\right\|_{H^{n}}+c_{n} \int_{0}^{t}\left\|U_{j}(s)\right\|_{H^{n}} \mathrm{~d} s
$$

where $c_{n}$ depends on $g$ and $\left\|u_{0}\right\|_{H^{n}}$ so that the proof can be concluded by Gronwall's lemma. Inequality (4.7) is obvious for all $j \in \mathbb{N}$ and $n=1$. We suppose that (4.7) is true up to the rank $n \geq 2$ and we consider $u_{0} \in H^{n+1}$. Because of Lemma 4.2 and Remark 4.3 there exists $C>0$ such that for all $j \in \mathbb{N}$,

$$
\begin{aligned}
\left|\partial_{x}^{n+1} g\left(U_{j}\right)\right| & \leq C \sum_{k=1}^{n+1}\left\|\nabla^{k} g\right\|_{\infty} \sum_{\substack{1 \leq l_{1} \leq \ldots \leq l_{k} \leq n \\
l_{1}+\ldots+l_{k}=n+1}} \prod_{r=1}^{k}\left|\partial_{x}^{l_{r}} U_{j}\right| \\
& \leq C\|\nabla g\|_{\infty}\left|\partial_{x}^{n+1} U_{j}\right|+C \sum_{k=2}^{n+1}\left\|\nabla^{k} g\right\|_{\infty} \sum_{\substack{1 \leq l_{1} \leq \ldots \leq l_{k} \leq n \\
l_{1}+\ldots+l_{k}=n+1}} \prod_{r=1}^{k}\left|\partial_{x}^{l_{r}} U_{j}\right|
\end{aligned}
$$

Using the Sobolev inequality $\|\phi\|_{L^{\infty}} \leq C\|\phi\|_{H^{1}}$, we get

$$
\begin{aligned}
\left\|\partial_{x}^{n+1} g\left(U_{j}\right)\right\|_{L^{2}} & \leq C\|\nabla g\|_{\infty}\left\|\partial_{x}^{n+1} U_{j}\right\|_{L^{2}}+C \sum_{k=2}^{n+1}\left\|\nabla^{k} g\right\|_{\infty} \sum_{\substack{1 \leq l_{1} \leq \ldots \leq l_{k} \leq n \\
l_{1}+\ldots+l_{k}=n+1}}\left\|\prod_{r=1}^{k} \partial_{x}^{l_{r}} U_{j}\right\|_{L^{2}} \\
& \leq C\|\nabla g\|_{\infty}\left\|\partial_{x}^{n+1} U_{j}\right\|_{L^{2}}+C \sum_{k=2}^{n+1}\left\|\nabla^{k} g\right\|_{\infty} \sum_{\substack{1 \leq l_{1} \leq \ldots \leq l_{k} \leq n \\
l_{1}+\ldots+l_{k}=n+1}}\left\|\partial_{x}^{l_{1}} U_{j}\right\|_{L^{2}} \prod_{r=2}^{k}\left\|\partial_{x}^{l_{r}} U_{j}\right\|_{L^{\infty}} \\
& \leq C\|\nabla g\|_{\infty}\left\|\partial_{x}^{n+1} U_{j}\right\|_{L^{2}}+C \sum_{k=2}^{n+1}\left\|\nabla^{k} g\right\|_{\infty} \sum_{\substack{1 \leq l_{1} \leq \ldots \leq l_{k} \leq n \\
l_{1}+\ldots+l_{k}=n+1}}\left\|U_{j}\right\|_{H^{l_{1}}} \prod_{r=2}^{k}\left\|U_{j}\right\|_{H^{l_{r}+1}}
\end{aligned}
$$


In the last sum on the set $\left\{1 \leq l_{1} \leq \ldots \leq l_{k} \leq n ; l_{1}+\ldots+l_{k}=n+1\right\}$, at most one index from $\left\{l_{1}, l_{2}+1, \ldots, l_{k}+1\right\}$ can be equal to $n+1$. Moreover, by assumption, we have $\sup _{\theta \in[0,1]}\left\|U_{j}(\theta)\right\|_{H^{l}} \leq C_{l}$ for every $l \in\{0, \ldots, n\}$. We then get that for all $t$,

$$
\left\|\partial_{x}^{n+1} U_{j+1}(t)\right\|_{L^{2}} \leq\left\|\partial_{x}^{n+1} u_{0}\right\|_{L^{2}}+K \int_{0}^{t}\left\|U_{j}(\theta)\right\|_{H^{n+1}} \mathrm{~d} \theta
$$

where $K$ depends on $g$ and $\left\|u_{0}\right\|_{H^{n+1}}$. Using (4.7), we prove by induction that

$$
\left\|U_{j+1}(t)\right\|_{H^{n+1}} \leq\left\|u_{0}\right\|_{H^{n+1}}+c_{n+1} \int_{0}^{t}\left\|U_{j}(\theta)\right\|_{H^{n+1}} \mathrm{~d} \theta,
$$

where $c_{n+1}$ depends on $g$ and $\left\|u_{0}\right\|_{H^{n+1}}$. This concludes the proof.

\subsection{Proof of Theorem 2.4}

We now state some lemmas for the proof of Theorem 2.4. The first lemma deals with the boundedness of the splitting operators $\left\{Z\left(t_{0}, t\right)\right\}_{t \in\left[t_{0}, 1\right]}$ in $H^{k}$ for $t_{0} \in[0,1[$ under some assumptions on $g$.

Lemma 4.7. Let $v_{0} \in L^{2}$. If there exists $k \in \mathbb{N}$ such that $v_{0} \in H^{k}, g$ is $k$ times differentiable and its derivatives up to the order $k$ are bounded, then there exists a (deterministic) constant $C_{\infty, k}>0$ such that for every $\left(t_{0}, t\right) \in[0,1]^{2}$ satisfying $t_{0}<t$ we have

$$
\max _{\theta \in\left[t_{0}, t\right]}\left\|Z\left(t_{0}, \theta\right) v_{0}\right\|_{H^{k}} \leq C_{\infty, k} \text { and } \max _{\theta \in\left[t_{0}, t\right]}\left\|g\left(Z\left(t_{0}, \theta\right) v_{0}\right)\right\|_{H^{k}} \leq C_{\infty, k}
$$

The constant $C_{\infty, k}$ depends only on $\left\|u_{0}\right\|_{H^{k}}$.

Proof. We follow the same lines as the proof of Lemma 4.5 (and Lem. 4.6).

The two following lemmas provide useful estimates on the operators $X$ and $Z$. Lemma 4.8 essentially states the continuity of $\left\{X\left(t_{0}, t\right)\right\}_{t \in\left[t_{0}, 1\right]}$ for $t_{0} \in[0,1[$.

Lemma 4.8. Let $v_{0} \in H^{\delta}$. There exists a constant $C_{P}$ only depending on $P$ such that for every $\left(t_{0}, t\right) \in[0,1]^{2}$ satisfying $t_{0}<t$ we have

$$
\left\|X\left(t_{0}, t\right) v_{0}-v_{0}\right\|_{L^{2}} \leq C_{P}\left|W(t)-W\left(t_{0}\right)\right|\left\|v_{0}\right\|_{H^{\delta}}
$$

Proof. Using the estimate $|\exp (i x)-1|^{2} \leq x^{2}$, we get

$$
\begin{aligned}
\left\|X\left(t_{0}, t\right) v_{0}-v_{0}\right\|_{L^{2}}^{2} & =\int_{-\infty}^{+\infty}\left|\mathcal{F}\left(v_{0}\right)(\theta) \exp \left(-i\left(W(t)-W\left(t_{0}\right)\right) P(\theta)\right)-\mathcal{F}\left(v_{0}\right)(\theta)\right|^{2} \mathrm{~d} \theta \\
& \leq\left(W(t)-W\left(t_{0}\right)\right)^{2} \int_{-\infty}^{+\infty}\left|P(\theta) \mathcal{F}\left(v_{0}\right)(\theta)\right|^{2} \mathrm{~d} \theta \\
& \leq C_{P}^{2}\left(W(t)-W\left(t_{0}\right)\right)^{2}\left\|v_{0}\right\|_{H^{\delta}}^{2}
\end{aligned}
$$

where, for instance, $C_{P}^{2}=\delta \sum_{j=0}^{\delta} p_{j}^{2}$.

Finally, the Lemma stated below proves the Lipschitz property of the operators $Z\left(t_{0}, t\right)$ (for $t_{0}<t$ ) from $L^{2}$ to itself. 
Lemma 4.9. Let $v_{0}, v_{1} \in L^{2}$. If there exists $k \in \mathbb{N}$ such that $v_{0}$ and $v_{1} \in H^{k}, g$ is $k$ times differentiable and its derivatives up to the order $k$ are bounded, then there exists a (deterministic) constant $C_{g}>0$ such that for every $\left(t_{0}, t\right) \in[0,1]^{2}$ satisfying $t_{0}<t$ we have

$$
\left\|Z\left(t_{0}, t\right) v_{0}-Z\left(t_{0}, t\right) v_{1}\right\|_{L^{2}} \leq\left\|v_{0}-v_{1}\right\|_{L^{2}} \exp \left(C_{g}\left(t-t_{0}\right)\right) .
$$

The constant $C_{g}$ depends only on $g$.

Proof. Because of (2.5), it is enough to prove that there exists a (deterministic) constant $C_{g}>0$ such that for every $\left(t_{0}, t\right) \in[0,1]^{2}$ satisfying $t_{0}<t$ we have

$$
\left\|Y\left(t-t_{0}\right) v_{0}-Y\left(t-t_{0}\right) v_{1}\right\|_{L^{2}} \leq\left\|v_{0}-v_{1}\right\|_{L^{2}} \exp \left(C_{g}\left(t-t_{0}\right)\right) .
$$

Since (2.7) and because $g$ is Lipschitz, we have

$$
\begin{aligned}
\left\|Y\left(t-t_{0}\right) v_{0}-Y\left(t-t_{0}\right) v_{1}\right\|_{L^{2}} & \leq\left\|v_{0}-v_{1}\right\|_{L^{2}}+\int_{t_{0}}^{t}\left\|g\left(Y\left(\theta-t_{0}\right) v_{0}\right)-g\left(Y\left(\theta-t_{0}\right) v_{1}\right)\right\|_{L^{2}} \mathrm{~d} \theta \\
& \leq\left\|v_{0}-v_{1}\right\|_{L^{2}}+\|g\|_{L i p} \int_{t_{0}}^{t}\left\|Y\left(\theta-t_{0}\right) v_{0}-Y\left(\theta-t_{0}\right) v_{1}\right\|_{L^{2}} \mathrm{~d} \theta .
\end{aligned}
$$

We conclude by Gronwall's lemma.

To prove Theorem 2.4 we deal with the local error of the scheme (Lem. 4.10) and then we prove the estimate of the global error.

Lemma 4.10. Let $v_{0} \in H^{\delta}$. There exists a (deterministic) constant $C=C\left(g,\left\|v_{0}\right\|_{H^{\delta}}\right)>0$ depending only on $g$ and $\left\|v_{0}\right\|_{H^{\delta}}$ such that for every $\left(t_{0}, t\right) \in[0,1]^{2}$ satisfying $t_{0}<t$ we have

$$
\left\|S\left(t_{0}, t\right) v_{0}-Y\left(t-t_{0}\right) X\left(t_{0}, t\right) v_{0}\right\|_{L^{2}} \leq C \mathcal{I}_{W}\left(t_{0}, t\right) .
$$

Proof. Throughout the proof, the letter $C$ stands for a deterministic constant, can vary from line to line and depends only on $P, g$ and $\left\|v_{0}\right\|_{H^{\delta}}$. First, we remark that

$$
Z\left(t_{0}, t\right) v_{0}=Y\left(t-t_{0}\right) X\left(t_{0}, t\right) v_{0}=X\left(t_{0}, t\right) v_{0}+\int_{t_{0}}^{t} g\left(Y\left(\theta-t_{0}\right) X\left(t_{0}, t\right) v_{0}\right) \mathrm{d} \theta .
$$

Thus, we have, using (2.6) and (4.9),

$$
S\left(t_{0}, t\right) v_{0}-Z\left(t_{0}, t\right) v_{0}=R_{1}\left(t_{0}, t\right)+R_{2}\left(t_{0}, t\right)+R_{3}\left(t_{0}, t\right)
$$

where

$$
\begin{aligned}
R_{1}\left(t_{0}, t\right) & =\int_{t_{0}}^{t} X(\theta, t)\left\{g\left(S\left(t_{0}, \theta\right) v_{0}\right)-g\left(Z\left(t_{0}, \theta\right) v_{0}\right)\right\} \mathrm{d} \theta \\
R_{2}\left(t_{0}, t\right) & =\int_{t_{0}}^{t} X(\theta, t) g\left(Z\left(t_{0}, \theta\right) v_{0}\right)-g\left(Z\left(t_{0}, \theta\right) v_{0}\right) \mathrm{d} \theta \\
R_{3}\left(t_{0}, t\right) & =\int_{t_{0}}^{t}\left(g\left(Z\left(t_{0}, \theta\right) v_{0}\right)-g\left(Y\left(\theta-t_{0}\right) X\left(t_{0}, t\right) v_{0}\right)\right) \mathrm{d} \theta \\
& =\int_{t_{0}}^{t}\left(g\left(Y\left(\theta-t_{0}\right) X\left(t_{0}, \theta\right) v_{0}\right)-g\left(Y\left(\theta-t_{0}\right) X\left(t_{0}, t\right) v_{0}\right)\right) \mathrm{d} \theta
\end{aligned}
$$


Because of (2.5), we have

$$
\left\|R_{1}\left(t_{0}, t\right)\right\|_{L^{2}} \leq\|g\|_{L i p} \int_{t_{0}}^{t}\left\|S\left(t_{0}, \theta\right) v_{0}-Z\left(t_{0}, \theta\right) v_{0}\right\|_{L^{2}} \mathrm{~d} \theta .
$$

Since Lemmas 4.8 and 4.7 we have

$$
\begin{aligned}
\left\|R_{2}\left(t_{0}, t\right)\right\|_{L^{2}} & \leq C \int_{t_{0}}^{t}|W(t)-W(\theta)|\left\|g\left(Z\left(z_{0}, z^{\prime}\right) v_{0}\right)\right\|_{L^{2}} \mathrm{~d} \theta \\
& \leq C\left(\int_{t_{0}}^{t}|W(t)-W(\theta)| \mathrm{d} \theta\right) \max _{s \in\left[t_{0}, t\right]}\left\|g\left(Z\left(t_{0}, s\right) v_{0}\right)\right\|_{L^{2}} .
\end{aligned}
$$

From Lemmas 4.8 and 4.9 we obtain that

$$
\begin{aligned}
\left\|R_{3}\left(t_{0}, t\right)\right\|_{L^{2}} & \leq \int_{t_{0}}^{t}\left\|g\left(Z\left(t_{0}, \theta\right) v_{0}\right)-g\left(Y\left(\theta-t_{0}\right) X\left(t_{0}, t\right) v_{0}\right)\right\|_{L^{2}} \mathrm{~d} \theta \\
& \leq C \int_{z_{0}}^{z}\left\|X\left(t_{0}, \theta\right) v_{0}-X\left(t_{0}, t\right) v_{0}\right\|_{L^{2}} \mathrm{~d} \theta \\
& \leq C\left(\int_{t_{0}}^{t}|W(t)-W(\theta)| \mathrm{d} \theta\right) .
\end{aligned}
$$

Then, by (4.10)-(4.12) and (4.13), for every $\left(t_{0}, t\right) \in[0,1]^{2}$ satisfying $t_{0}<t$ we get

$$
\left\|S\left(t_{0}, t\right) v_{0}-Z\left(t_{0}, t\right) v_{0}\right\|_{L^{2}} \leq C \int_{t_{0}}^{t}|W(t)-W(\theta)| \mathrm{d} \theta+\|g\|_{L i p} \int_{t_{0}}^{t}\left\|S\left(t_{0}, \theta\right) v_{0}-Z\left(t_{0}, \theta\right) v_{0}\right\|_{L^{2}} \mathrm{~d} \theta .
$$

We complete the proof by using the modified Gronwall lemma recalled below (Lem. 4.11).

Lemma 4.11 (Modified Gronwall lemma). Let $\phi$ and $f$ be two nonnegative functions defined on an interval $[a, b]$. We assume that there exists a constant $c>0$ such that for every $t \in[a, b], \phi(t) \leq f(t)+c \int_{a}^{t} \phi(\theta) \mathrm{d} \theta$. Then, for every $t \in[a, b], \phi(t) \leq f(t)+c \mathrm{e}^{c t} \int_{a}^{t} \mathrm{e}^{-c \theta} f(\theta) \mathrm{d} \theta$.

Remark 4.12. Remark that we do not assume $f$ to be increasing.

Now we prove Theorem 2.4.

Proof of Theorem 2.4. We write

$$
u_{n}^{h}-u(n h, \cdot)=\sum_{j=1}^{n}\left(Z^{n, h} \ldots Z^{j, h} S^{j-1, h} \ldots S^{1, h} u_{0}-Z^{n, h} \ldots Z^{j+1, h} S^{j, h} \ldots S_{1}^{h} u_{0}\right) .
$$

From Lemma 4.9, there exists a (deterministic) constant $C_{g}>0$ depending only on $g$ such that

$$
\left\|u^{n, h}-u(n h, \cdot)\right\|_{L^{2}} \leq \sum_{j=1}^{n} \mathrm{e}^{C_{g}(n-j)}\left\|\left(Z^{j, h}-S^{j, h}\right) S^{j-1, h} \ldots S^{1, h} u_{0}\right\|_{L^{2}} .
$$

By Lemmas 4.10 and 2.3 , there exists a (deterministic) constant $C\left(g,\left\|u_{0}\right\|_{H^{\delta}}\right)>0$ depending only on $g$ and $\left\|u_{0}\right\|_{H^{\delta}}$ such that

$$
\max _{n \in\{1, \ldots, N\}}\left\|u^{n, h}-u(n h, \cdot)\right\|_{L^{2}} \leq C\left(g,\left\|u_{0}\right\|_{H^{\delta}}\right) \sum_{j=1}^{N} \mathcal{I}_{W}((j-1) h, j h) .
$$

This concludes the proof. 


\subsection{Proof of the asymptotic-preserving property}

We first prove Theorem 2.8 which deals with the continuity of the solution with respect to $W$.

Proof of Theorem 2.8. Let $w_{1}$ and $w_{2}$ in $\mathcal{C}([0,1], \mathbb{R})$. We have

$$
\begin{aligned}
u_{w_{1}}(t, x)-u_{w_{2}}(t, x)= & X_{w_{1}}(0, t) u_{0}(x)-X_{w_{2}}(0, t) u_{0}(x)+\int_{0}^{t}\left(X_{w_{1}}(\theta, t)-X_{w_{2}}(\theta, t)\right) g\left(u_{w_{1}}(\theta, x)\right) \mathrm{d} \theta \\
& +\int_{0}^{t} X_{w_{2}}(\theta, t)\left(g\left(u_{w_{1}}(\theta, x)\right)-g\left(u_{w_{2}}(\theta, x)\right)\right) \mathrm{d} \theta .
\end{aligned}
$$

Taking the $L^{2}-$ norm, we get

$$
\begin{aligned}
\left\|u_{w_{1}}(t)-u_{w_{2}}(t)\right\|_{L^{2}} \leq & \left\|X_{w_{1}-w_{2}}(0, t) u_{0}-u_{0}\right\|_{L^{2}}+\int_{0}^{t}\left\|\left(X_{w_{1}}(\theta, t)-X_{w_{2}}(\theta, t)\right) g\left(u_{w_{1}}(\theta)\right)\right\|_{L^{2}} \mathrm{~d} \theta \\
& +\int_{0}^{t}\left\|X_{w_{2}}(\theta, t)\left(g\left(u_{w_{1}}(\theta)\right)-g\left(u_{w_{2}}(\theta)\right)\right)\right\|_{L^{2}} \mathrm{~d} \theta
\end{aligned}
$$

From Lemma 4.8, we get

$$
\begin{gathered}
\left\|X_{w_{1}}(0, t) u_{0}-X_{w_{2}}(0, t) u_{0}\right\|_{L^{2}}+\int_{0}^{t}\left\|\left(X_{w_{1}}(\theta, t)-X_{w_{2}}(\theta, t)\right) g\left(u_{w_{1}}(\theta)\right)\right\|_{L^{2}} \mathrm{~d} \theta \\
\quad=\left\|X_{w_{1}}(0, t) u_{0}-X_{w_{2}}(0, t) u_{0}\right\|_{L^{2}}+\int_{0}^{t}\left\|X_{w_{1}-w_{2}}(\theta, t) g\left(u_{w_{1}}(\theta)\right)-g\left(u_{w_{1}}(\theta)\right)\right\|_{L^{2}} \mathrm{~d} \theta \\
\leq\left\|w_{1}-w_{2}\right\|_{\infty}\left(\left\|u_{0}\right\|_{H^{\delta}}+2 \int_{0}^{t}\left\|g\left(u_{w_{1}}(\theta)\right)\right\|_{H^{\delta}} \mathrm{d} \theta\right) \leq C\left\|w_{1}-w_{2}\right\|_{\infty}
\end{gathered}
$$

where the constant $C>0$ only depends on $g$ and $\left\|u_{0}\right\|_{H^{\delta}}$. Moreover,

$$
\begin{aligned}
\int_{0}^{t}\left\|X_{w_{2}}(\theta, t)\left(g\left(u_{w_{1}}(\theta)\right)-g\left(u_{w_{2}}(\theta)\right)\right)\right\|_{L^{2}} \mathrm{~d} \theta & =\int_{0}^{t}\left\|g\left(u_{w_{1}}(\theta)\right)-g\left(u_{w_{2}}(\theta)\right)\right\|_{L^{2}} \mathrm{~d} \theta \\
& \leq\|\nabla g\|_{\infty} \int_{0}^{t}\left\|u_{w_{1}}(\theta)-u_{w_{2}}(\theta)\right\|_{L^{2}} \mathrm{~d} \theta .
\end{aligned}
$$

Then, we obtain

$$
\left\|u_{w_{1}}(t)-u_{w_{2}}(t)\right\|_{L^{2}} \leq C\left\|w_{1}-w_{2}\right\|_{\infty}+\|\nabla g\|_{\infty} \int_{0}^{t}\left\|u_{w_{1}}(\theta)-u_{w_{2}}(\theta)\right\|_{L^{2}} \mathrm{~d} \theta .
$$

By Gronwall's lemma,

$$
\sup _{t \in[0,1]}\left\|u_{w_{1}}(t)-u_{w_{2}}(t)\right\|_{L^{2}} \leq C \exp \left(\|\nabla g\|_{\infty}\right)\left\|w_{1}-w_{2}\right\|_{\infty}
$$

which concludes the proof.

We then prove Theorem 2.9 which states the continuity of the splitting scheme with respect to $W$.

Proof of Theorem 2.9. By induction, it is enough to show that there exists $C>0$ such for all $j=1, \ldots, N$ and $\left(w_{1}, w_{2}\right)$ in $\mathcal{C}([0,1], \mathbb{R})^{2}$,

$$
\left\|u_{w_{1}}^{j, h}-u_{w_{2}}^{j, h}\right\|_{L^{2}} \leq C\left\|u_{w_{1}}^{j-1, h}-u_{w_{2}}^{j-1, h}\right\|_{L^{2}}+C\left\|w_{1}-w_{2}\right\|_{\infty} .
$$


Throughout this proof, $C$ stands for a positive constant which depends on $\left\|u_{0}\right\|_{H^{\delta}}, g$ and $N$ and can vary from line to line. We have, using (4.9),

$$
\begin{aligned}
u_{w_{1}}^{j, h}-u_{w_{2}}^{j, h}= & X_{w_{1}}^{j, h} u_{w_{1}}^{j-1, h}-X_{w_{2}}^{j, h} u_{w_{2}}^{j-1, h} \\
& +\int_{(j-1) h}^{j h}\left(g\left(Y(\theta-(j-1) h) X_{w_{1}}^{j, h} u_{w_{1}}^{j-1, h}\right)-g\left(Y(\theta-(j-1) h) X_{w_{2}}^{j, h} u_{w_{2}}^{j-1, h}\right)\right) \mathrm{d} \theta .
\end{aligned}
$$

Taking the $L^{2}$-norm, because $g$ is Lipschitz and using (4.8), we get

$$
\begin{aligned}
\left\|u_{w_{1}}^{j, h}-u_{w_{2}}^{j, h}\right\|_{L^{2}} \leq & \left\|X_{w_{1}}^{j, h} u_{w_{1}}^{j-1, h}-X_{w_{2}}^{j, h} u_{w_{2}}^{j-1, h}\right\|_{L^{2}} \\
& +\int_{(j-1) h}^{j h}\left\|g\left(Y(\theta-(j-1) h) X_{w_{1}}^{j, h} u_{w_{1}}^{j-1, h}\right)-g\left(Y(\theta-(j-1) h) X_{w_{2}}^{j, h} u_{w_{2}}^{j-1, h}\right)\right\|_{L^{2}} \mathrm{~d} \theta . \\
\leq & C\left\|X_{w_{1}}^{j, h} u_{w_{1}}^{j-1, h}-X_{w_{2}}^{j, h} u_{w_{2}}^{j-1, h}\right\|_{L^{2}} .
\end{aligned}
$$

Because of Lemmas 4.5 and 4.8, proceeding as in the proof of Theorem 2.8, we obtain

$$
\begin{aligned}
\left\|u_{w_{1}}^{j, h}-u_{w_{2}}^{j, h}\right\|_{L^{2}} & \leq C\left\|X_{w_{1}}^{j, h}\left(u_{w_{1}}^{j-1, h}-u_{w_{2}}^{j-1, h}\right)\right\|_{L^{2}}+C\left\|X_{w_{1}}^{j, h} u_{w_{2}}^{j-1, h}-X_{w_{2}}^{j, h} u_{w_{2}}^{j-1, h}\right\|_{L^{2}} \\
& \leq C\left\|u_{w_{1}}^{j-1, h}-u_{w_{2}}^{j-1, h}\right\|_{L^{2}}+C\left\|w_{1}-w_{2}\right\|_{\infty} .
\end{aligned}
$$

This concludes the proof.

Finally we prove Theorem 2.9 which establishes the uniform convergence of the splitting scheme when the time step goes to 0 .

Proof of Theorem 2.10. By Theorem 2.4 and Remark 2.5, there exists a constant $C$ which depends only on $g$ and $\left\|u_{0}\right\|_{H^{\delta}}$, such that for every $h \in(0,1]$ and every $\varepsilon \geq 0$,

$$
\mathbb{E}\left[\max _{n \in\{1, \ldots, N\}}\left\|u_{W^{\varepsilon}}^{n, h}-u_{W^{\varepsilon}}(n h, \cdot)\right\|_{L^{2}}\right] \leq C \sum_{n=1}^{N} \mathbb{E}\left[\mathcal{I}_{W^{\varepsilon}}((n-1) h, n h)\right] .
$$

By Hölder's inequality and (2.13), we have

$$
\begin{aligned}
\mathbb{E}\left[\mathcal{I}_{W^{\varepsilon}}((n-1) h, n h)\right] & \leq \int_{(n-1) h}^{n h}\left(\mathbb{E}\left[\left|W^{\varepsilon}(n h)-W^{\varepsilon}(\theta)\right|^{\gamma}\right]^{1 / \gamma}+\int_{(n-1) h}^{\theta} \mathbb{E}\left[\left|W^{\varepsilon}(\theta)-W^{\varepsilon}(\sigma)\right|^{\gamma}\right]^{1 / \gamma} \mathrm{d} \sigma\right) \mathrm{d} \theta \\
& \leq C^{\prime} h^{1+\beta / \gamma}
\end{aligned}
$$

where $C^{\prime}$ is a constant. Combining the last inequality with (4.15) we conclude the proof.

\section{REFERENCES}

[1] G.P. Agrawal, Nonlinear Fiber Optics, 3rd edition. Academic Press, San Diego (2001).

[2] G. Bal and L. Ryzhik, Time splitting for wave equations in random media. ESAIM: M2AN 38 (2004) 961-988

[3] G. Bal and L. Ryzhik, Time splitting for the Liouville equation in a random medium. Commun. Math. Sci. 2 (2004) 515-534.

[4] W. Bao, S. Jin and P.A. Markowich, On time-splitting spectral approximations for the Schrödinger equation in the semiclassical regime. J. Comput. Phys. 175 (2002) 487-524.

[5] A. Benassi, S. Jaffard and D. Roux, Gaussian processes and Pseudodifferential Elliptic operators. Rev. Math. Iberoam. 13 (1997) 19-89.

[6] C. Besse, B. Bidégaray and S. Descombes, Order estimates in time of splitting methods for the nonlinear Schrödinger equation. SIAM J. Numer. Anal. 40 (2002) 26-40.

[7] C. Besse, R. Carles and F. Mehats, An asymptotic preserving scheme based on a new formulation for NLS in the semiclassical limit. Multiscale Model. Simul. 11 (2013) 1228-1260.

[8] P. Billingsley, Convergence of Probability Measures. Wiley (1968). 
[9] S. Cohen and R. Marty, Invariance principle, multifractional Gaussian processes and long-range dependence. Ann. Inst. Henri Poincaré Probab. Stat. 44 (2008) 475-489.

[10] F. Coron and B. Perthame, Numerical passage from kinetic to fluid equations. SIAM J. Numer. Anal. 28 (1991) $26-42$.

[11] A. De Bouard and A. Debussche, The nonlinear Schrodinger equation with white noise dispersion. J. Functional Anal. 259 (2010) 1300-1321.

[12] A. Debussche and Y. Tsutsumi, 1D quintic nonlinear equation with white noise dispersion. J. Math. Pures Appl.96 (2011) 363-376.

[13] P. Degond, Asymptotic-Preserving Schemes for Fluid Models of Plasmas. Panoramas et Synthèses 39-40 (2013) 1-90.

[14] R.L. Dobrushin. Gaussian and their Subordinated Self-Similar Random Generalized Fields. Ann. Probab. 7 (1979) 1-28.

[15] R.L. Dobrushin and P. Major, Non-central limit theorems for nonlinear functionals of Gaussian fields. Z. Wahrsch. Verw. Gebiete $\mathbf{5 0}$ (1979) 27-52.

[16] P. Donnat, Quelques contributions mathématiques à l'optique non-linéaire. Ph.D. thesis, École Polytechnique (1993).

[17] S.N. Ethier and T.G. Kurtz, Markov processes, characterization and convergence. Wiley, New York (1986).

[18] J.-P. Fouque, J. Garnier, G. Papanicolaou and K. Solna, Wave Propagation and Time Reversal in Randomly Layered Media. Springer (2007).

[19] E. Gabetta, L. Pareschi and G. Toscani, Relaxation schemes for nonlinear kinetic equations SIAM J. Numer. Anal. 34 (1997) 2168-2194.

[20] J. Garnier and K. Solna, Pulse propagation in random media with long range correlation. Multiscale Model. Simul. 7 (2009) $1302-1324$.

[21] C. Gomez and O. Pinaud. Asymptotics of a time-splitting scheme for the random Schrödinger equation with long-range correlations. Math. Model. Numer. Anal. 48 (2014) 411-431.

[22] K. Itô. Multiple Wiener integral. J. Math. Soc. Jpn 3 (1951) 157-169

[23] S. Jin, Efficient Asymptotic-Preserving (AP) Schemes For Some Multiscale Kinetic Equations. SIAM J. Sci. Comput. 21 (1999) 441-454.

[24] S. Jin, P. Markowich and C. Sparber, Mathematical and computational methods for semiclassical Schrödinger equations. Acta Numer. 20 (2011) 121-209.

[25] A. Klar, An asymptotic-induced scheme for nonstationary transport equations in the diffusive limit. SIAM J. Numer. Anal. 35 (1998) 1073-1094.

[26] R. Marty, Asymptotic behavior of differential equations driven by periodic and random processes with slowly decaying correlations. ESAIM: PS 9 (2005) 165-184.

[27] R. Marty, On a splitting scheme for the nonlinear Schrödinger equation in a random medium. Commun. Math. Sci. 4 (2006) 679-705.

[28] R. Marty, From Hermite polynomials to multifractional processes. J. Appl. Prob. 50 (2013) 323-343.

[29] R. Marty and K. Solna, A general framework for waves in random media with long-range correlations. Ann. Appl. Probab. 21 (2011) 115-139.

[30] R.F. Peltier and J. Lévy Véhel, Multifractional Brownian motion: definition and preliminary results. preprint available at http://hal.inria.fr/inria-00074045/ (1995).

[31] G. Samorodnitsky and M.S. Taqqu, Stable non-Gaussian random processes. Chapman and Hall (1994).

[32] G. Strang, On the construction and comparison of difference schemes. SIAM J. Numer. Anal. 5 (1968) 506-517.

[33] M.S. Taqqu. Weak convergence to fractional Brownian motion and to the Rosenblatt process. Z. Wahrsch. Verw. Gebiete. 31 (1975) 287-302.

[34] M.S. Taqqu. Convergence of integrated processes of arbitrary Hermite rank. Z. Wahrsch. Verw. Gebiete 50 (1979) 53-83. 